\title{
The Liège Intranuclear Cascade model - Towards a unified description of nuclear reactions induced by nucleons and light ions from a few MeV to a few $\mathrm{GeV}$
}

\author{
Joseph Cugnon $^{1 \mathrm{a}}$, Alain Boudard ${ }^{2}$, Jean-Christophe David ${ }^{2}$, Sylvie Leray ${ }^{2}$, and Davide Mancusi ${ }^{2}$ \\ ${ }^{1}$ University of Liège, allée du 6 août 17, Bât. B5, B-4000 Liège 1, Belgium \\ ${ }^{2}$ CEA/Saclay, Irfu/SPhN, 91191 Gif-sur-Yvette Cedex, France
}

\begin{abstract}
The predictive power of the last version INCL4.6 of the Liège Intranuclear Cascade model for spallation is reviewed. The good results obtained both at low and high energy extend the domain of validity of the model and allow the description of spallation reactions, except the coherent processes, by a unique model from a few $\mathrm{MeV}$ to a few $\mathrm{GeV}$ incident energy.
\end{abstract}

\section{Introduction}

Spallation reactions are usually defined as the reactions induced by hadrons and/or light ions with an incident energy comprised between, say $100 \mathrm{MeV}$ to $2 \mathrm{GeV}$ (per nucleon for incident light ions). They are characterized by a copious emission of neutrons, accompanied by a lesser rate emission of light charged particles (lcp's). They can give rise to an extended range (in mass and charge) of target residues. The renewed interest for spallation reactions is testified by several research programs, conducted especially in Europe, aiming at improving theoretical models and at producing high quality data in order to benchmark the former. This interest is fed by the development of various applications, connected to both fundamental and applied physics: transmutation of nuclear waste in acceleratordriven systems, neutron spallation sources, protection against radiation in manned space missions, cancer hadrontherapy, shielding of accelerators and detectors, production of radioactive beams, interaction of cosmic rays, etc (see Ref.[1] for illustrative references). It is now widely accepted that spallation reactions can be described by a two-step process: an intranuclear cascade stage emitting rapid particles, followed by the de-excitation of a target remnant.

The purpose of the present paper is twofold. The first one is to present the Liège intranuclear cascade (INCL4) model for spallation reactions, its latest developments and its predictive power. The second one is to show that the results obtained with this model, coupled to the ABLA07 de-excitation model[2], point towards the possibility of having a unified description of nuclear reactions induced by nucleons and light ions, extending from a few $\mathrm{MeV}$ (above the so-called resonance region) to several $\mathrm{GeV}$ (per nucleon for light ions). By this, we mean that a single model, with fixed parameters, is able to describe satisfactorily most of the available data in this range. This, of course, excludes coherent reaction processes, such as elastic scattering and coherent excitation of the target to low-lying states.

a Corresponding author: J.Cugnon@ulg.ac.be 


\section{Short description of the INCL4 model}

In order to exhibit the implications of the last developments, we put this description in some historical perspective. The original model for nucleon-induced reactions (INCL4.2) is described in Ref.[3]. We simply recall the main features here. The fate of all particles is followed in phase space and the reaction process can be viewed as a sequence of binary collisions occuring as in free space, except that there are subject to the Pauli blocking, operating also in phase space. This model is free of adjustable parameters. In particular -and this is a unique feature of INCL4.2- the stopping time is determined self-consistently by the model itself. In this version, incident light particles (up to alpha's) are viewed as a collection of on-shell nucleons, with a Fermi motion inside their reference frame, and with a total energy equal to the nominal total incident energy.

In 2009, an upgraded version (INCL4.5) was released[4]. Two main new features are introduced. First, lcp's can be emitted by the cascade owing to a dynamical coalescence model: nucleons leaving the target may carry other nucleons provided they are sufficiently close by in phase space. Second, the behaviour of the model at low incident energy is improved, mainly by a better account of soft collisions, especially in the first instances of the reaction process. In INCL4.2, these soft collisions, which are of little importance for the energy-momentum flow, were basically neglected. Taking them into account considerably enhances the probability of the first collision for the projectile, at low energy, and thus the reaction cross section, while the spectrum of emitted particles is barely affected. See Refs.[1,4] for more detail. These modifications imply the introduction of adjustable parameters, which, however, have been fixed once for all.

Finally, the last version of the model, INCL4.6, has been published in 2013. A detailed account can be found in Ref.[1]. The main new development involves the treatment of cluster-induced reactions. The incident cluster is viewed as a collection of off-shell nucleons - their energy is reduced by an average potential energy- in order to have correct energy and momentum of the projectile. The projectile nucleons are divided into geometrical spectators (those which do not intercept the target) and participants. The former are put on shell as free nucleons or bound to clusters. The latter are making a compound nucleus or are initiating in common a usual cascade process, according as their available energy is low or high. The details of the procedure are given in Ref.[1].

The features of the ABLA07 model are given in Ref.[2].

\section{Results}

\subsection{Nucleon-induced reactions}

We do not have the place to discuss all the results here. A good account can be found in Ref.[1] and in the intercomparison of 17 spallation models organized by the IAEA[5]. The latter has shown that the INCl4.6-ABLA07 combined model is one of the best for all kinds of observable quantities. This is illustrated in Fig. 1 for the residue cross sections.

Among the most remarkable achievements of our model, we want to point out its predictions at low and high energy. The predictions for the total reaction cross sections are very good down to 10 $\mathrm{MeV}$ [1]. This result, which is a test of the cascade alone, is due to a better treatment of the soft collisions, as alluded to above. We also obtained good results for particle spectra for reactions at few tens of $\mathrm{MeV}$, well below the alleged lower limit of validity of cascade models -say, $200 \mathrm{MeV}$. Tentative explanations of this paradoxical situation can be found in Refs.[6,7]. A typical result is shown in Fig.2. As an illustration of our good results at high energy, we single out the satisfactory reproduction of the pion production measurements of the HARP collaboration[8], up to $12 \mathrm{GeV}$, as extensively discussed in Ref.[9]. 


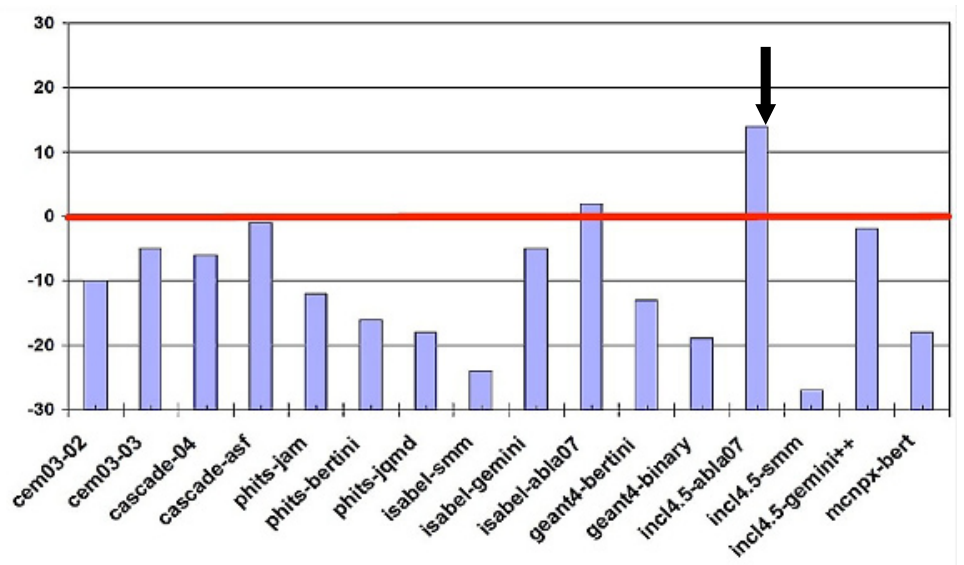

Figure 1. Comparison of the quality factors obtained by various spallation models for a set of residue cross sections. See Ref.[4] for definition of the quality factor and details. Our model is singled out by the arrow. Adapted from Ref.[4].

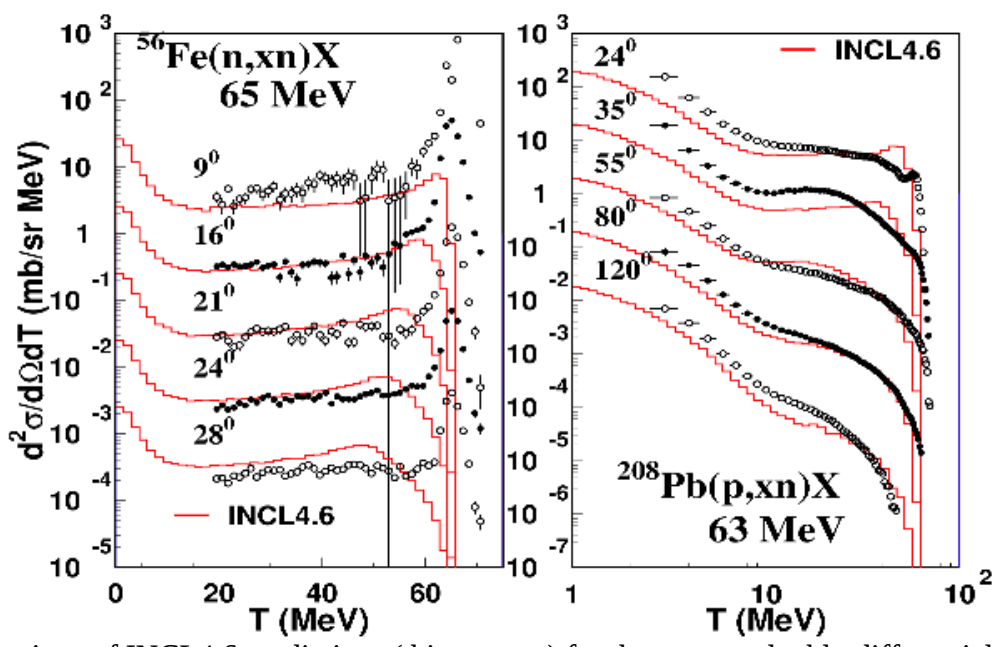

Figure 2. Comparison of INCL4.6 predictions ( histograms) for the neutron double-differential cross sections in $n$ $+{ }^{56} \mathrm{Fe}$ collisions at $65 \mathrm{MeV}$ (left panel, data from Ref. [10]) and in $\mathrm{p}+{ }^{208} \mathrm{~Pb}$ collisions at $63 \mathrm{MeV}$ (right panel, data from Ref. [11]), as functions of the emitted neutron kinetic energy $\mathrm{T}$. The various spectra have been multiplied by $10^{0}, 10^{-1}, 10^{-2}$, etc, for increasing angles, starting from the smallest angle. Adapted from Ref.[1].

\subsection{Cluster-induced reactions}

For light-cluster induced reactions, say up to $A=4$, we obtained good results for total reaction cross sections and for individual residue cross sections, even at low energy. This is demonstrated in Fig. 3 below. The model could not yet be evaluated seriously for double differential particle cross sections, because of the scarcity of experimental data. On the other hand, it has been tested successfully for the astatine production off a lead-bismuth target irradiated by a proton beam at 1 and $1.4 \mathrm{GeV}$ [12]. To obtain a good agreement with the data, it is necessary to have the correct ( $a, x n$ ) cross sections for the secondary a-induced reactions on ${ }^{209} \mathrm{Bi}$. This is explained at length in Ref. [13]. The model has also been tested for heavier ions, like $\mathrm{C}$ or $\mathrm{O}$, on data related to hadrontherapy, involving projectile fragmentation, but also neutron and light charged particle spectra. Satisfactory results are obtained in these cases, especially concerning the residue production in bombardment of a PMMA target by a 95A 
$\mathrm{MeV}{ }^{12} \mathrm{C}$ beam[15] and neutron spectra in irradiation of a water target by $200 \mathrm{MeV}{ }^{12} \mathrm{C}$ ions[16]. Details can be found in Ref.[17]

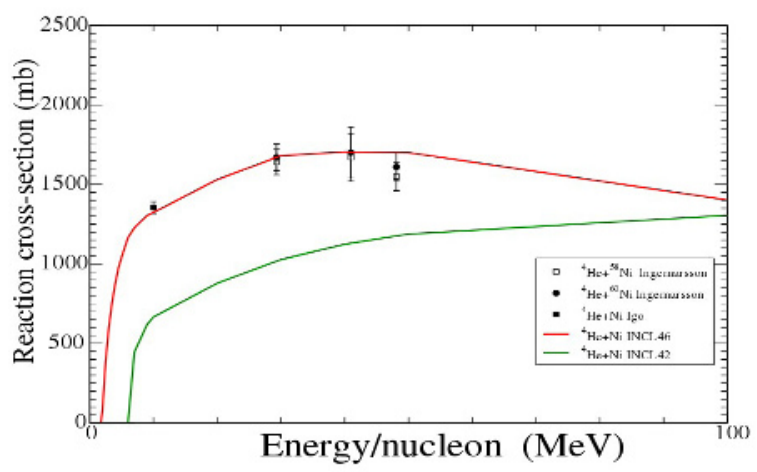

Figure 3. Comparison of the INCL4.6 (upper curve) and INCL4.2 (lower curve) for the total ${ }^{4} \mathrm{He}$ reaction cross section on ${ }^{58} \mathrm{Ni} /{ }^{60} \mathrm{Ni}$ with the experimental data of Ref.[14]. Adapted from Ref.[1].

\section{References}

1. A. Boudard, J. Cugnon, J.-C. David, S. Leray, D. Mancusi, Phys. Rev. C 87, 014606 (2013)

2. A. Kelić, M. V. Ricciardi and K.-H. Schmidt, in "Joint ICTP-IAEA Advanced Workshop on Model Codes for Spallation Reactions”, ed. by D. Filges et al., IAEA, INDC (NDS)-1530, (IAEA Publications, Vienna, 2008) pp.181-222.

3. A. Boudard, J. Cugnon, S. Leray, C. Volant, Phys. Rev. C 66, 044615 (2002)

4. J. Cugnon, A. Boudard, S. Leray, D. Mancusi, Proc. of Int. Topical Meeting on Nuclear Research Applications and Utilization of Accelerators (AccApp09), IAEA, Vienna, 2009 (IAEA Publications, Vienna, 2010), ISBN 978-92-0-150410-4, SM/SR-02, 8p.

5. IAEA Benchmark of Spallation Models, http://www-nds.iaea.org/spallations

6. Y. Yariv et al, in Proceedings of the International Conference on Nuclear Data for Science and Technology, April 22-27, 2007, ed. by O. Bersillon et al (EDP Sciences, Les Ulis, 2008) p. 296

7. J. Cugnon, Few-Body Systems Vol.53, Nos.1-2, 143 (2012)

8. HARP Collaboration, Eur. Phys. J. C 54, 37 (2008)

9. S. Pedoux, J. Cugnon, Nucl. Phys. A866, 16 (2011)

10. E. L. Hjort et al., Phys. Rev. C 53, 237 (1996)

11. A. Guertin et al., Eur. Phys. J. A 23, 49 (2005)

12. Y. Tall et al., Proceedings of the International Conference on Nuclear Data for Science and Technology, April 22-27, 2007, ed. by O. Bersillon et al (EDP Sciences, Les Ulis, 2008) p.1069.

13. J.-C. David, A. Boudard, J. Cugnon, S. Ghali, S. Leray, D. Mancusi, L. Zanini, Eur. Phys. J. A 49, 29 (2013)

14. A. Ingemarsson et al., Nucl. Phys. A676, 3 (2000), Nucl. Phys. A696, 3 (2001); G. Igo, B. D. Wilkins, Phys. Rev. 131, 1251 (1963)

15. B. Braunn et al., Nucl. Inst. and Meth. B269, 2676 (2011)

16. K. Gunzert-Marx et al. New Journal of Phys. 10, 075003 (2008)

17. S. Leray et al, Proceedings of the SATIF11 workshop, OECD, to be published 